\title{
PARTNER SELECTION WITH NETWORK INTERDEPENDENCIES: AN APPLICATION
}

\author{
Toni Jarimo and Iiro Salkari \\ VTT Technical Research Centre of Finland \\ toni.jarimo@vtt.fi, iiro.salkari@vtt.fi,FINLAND \\ Stefan Bollhalter \\ Virtuelle Fabrik $A G$ \\ stefan.bollhalter@virtuelle-fabrik.com,SWITZERLAND
}

This paper describes a virtual organization (VO) partner-selection case of Virtuelle Fabrik $A G$, which is an operating virtual organization breeding environment (VBE). We approach the partner-selection problem through a recently developed optimization framework; therefore we also study the applicability of the framework. The results suggest that an optimization framework is potentially useful for supporting VO creation.

\section{INTRODUCTION}

Collaborative networks are an increasing trend both in global and regional business. As individual companies seek efficiency by focusing on core competences and outsourcing other operations, the degree of inter-firm transactions grows. Simultaneously, the efficient management of network relations becomes a condition of survival. Therefore, models that support the management of network relations are called for. (E.g. Gulati et al. 2000, Camarinha-Matos and Afsarmanesh 2005)

A specific problem of collaborative networks is partner selection. On one hand, partner selection is a matching problem between the needs of the customer and the competences of partner candidates. On the other hand, it is a portfolio selection problem, where the consideration of the whole is relevant. The reason for this is that different network interdependencies between partner candidates may well affect the efficiency of the whole, which makes it insufficient to select partners one by one (e.g. Degraeve et al. 2000). Examples of such interdependencies are collaboration history, geographical locations, and ICT infrastructure (Camarinha-Matos and Afsarmanesh 2003). Accounting for network interdependencies has been empowered through the emergence virtual organization breeding environments (VBE, Camarinha-Matos and Afsarmanesh 2003), which consist of memberorganizations that have developed a mutually agreed cooperation structure for the 
creation of virtual organizations (see also Lau and Wong 2001, Tølle and Bernus 2003).

In this paper, we apply our recently developed partner selection framework (see, Jarimo and Pulkkinen 2005, Jarimo and Salo 2006) to a real-life case of an existing VBE, the Virtuelle Fabrik AG. The partner selection framework uses multi-criteria optimisation techniques to identify Pareto-efficient network configurations, which the decision-maker (DM) can manually compare.

The rest of this paper is structured as follows. Section 2 briefly reviews the partner selection framework. Section 3 analyses the case and Section 4 concludes.

\section{OPTIMISATION FRAMEWORK FOR VO PARTNER SELECTION}

This section briefly reviews the model used in the application this paper presents. The model is described in more detail in Jarimo and Pulkkinen (2005) and Jarimo and Salo (2006).

\subsection{Purpose of the model}

Our model finds and evaluates feasible VO configurations with respect to userdefined criteria. The problem is formulated as that of work-allocation, where the feasibility of a configuration is defined through its ability to perform a project. The project is described by tasks, each of which requires a specific competence. In addition, work-loads (e.g. person month) can be attached to the tasks.

The model accounts for a large variety of selection criteria. First, total costs include fixed and variable work costs and transportation costs. Second, stochastic risk measures are applied to model risks of failure, delays, or capacity shortfall. Third, network interdependencies, such as collaboration history or total number of partners can be taken into account.

Using the model, a decision-maker (DM) can identify a set of Pareto-optimal configurations, of which the DM can manually select the preferred one. In other words, the model suggests several alternatives that are "good" in respect of different preferences over the selection criteria. Hence, the DM does not need to explicitly weight the criteria, but instead can identify configurations that reflect different preferences.

\subsection{Approach}

The model formulates the VO partner selection as a work-allocation problem, which is approached by multi-objective mixed integer linear programming (MILP). The benefit of MILP models is that they can be readily solved using well known algorithms. Indeed, computational experiments suggest that our MILP models are tractable for problems of reasonable size and consequently potentially useful for VO decision making.

The multi-objectivity is captured by goal-programming techniques (e.g. Taha, 1997) or additive value functions (MAVT, Keeney and Raiffa, 1993). Heuristic algorithms are used to find Pareto-efficient solutions. 


\subsection{Inputs and Outputs}

The inputs of the model are as follows. There are $n$ tasks with a work-load (e.g. person month) attached to each of them. The tasks also have a relational work sequence, and information on possible transportation needs between tasks. For each task $j$, there are $m_{j}$ partner candidates, the total number of candidates being $m=\Sigma_{j}$ $m_{j}$. Each candidate has a capacity, and fixed and variable cost for working on the tasks to which it is a candidate. This data is easily represented by matrices, where the rows and columns represent the candidates and the tasks. A candidate can have a probability distribution over its capacities, reflecting the uncertainty on true capacity. Moreover, each candidate can have a fixed cost for working on the project, a geographical location, collaboration history with other candidates, etc.

The DM needs to define the selection criteria for partner selection. The problem is to select a good VO configuration for the project, subject to the above information on the project and candidate partners. This problem is essentially that of allocating the task workloads to partners, in recognition of their capacities and the decision criteria that are relevant to the evaluation of alternative partner configurations.

The output of the model is a set of Pareto-efficient work allocations, i.e. VO configurations. The constituents of this set depends on how the DM has expressed his/her preferences over the selection criteria. If the DM provides with perfect information on the relative importance of the selection criteria, a unique Paretoefficient configuration is suggested. Again, if the preference information is imperfect, possibly multiple configurations are identified. The DM can then manually select the most preferred configuration.

The scores of the identified configurations on the selection criteria are also given. Hence, in addition to the partners in Pareto-efficient configurations, the DM can compare the expected performance of the configurations.

\section{APPLICATION: MAGNETIC CLUTCH PROTOTYPE FOR TRUCKS}

We applied the model to a Virtuelle Fabrik partner selection case, which is described in this section. The results suggest that relevant criteria can be taken into account and reasonable configurations are identified.

\subsection{Project Description}

The aim of the project was to construct a prototype magnetic clutch to be used in trucks. The project was broken down into nine tasks, which were 1) Grinding, 2) Gear milling, 3) Metal sheet forming, 4) Milling and turning of bigger parts, 5) Welding, 6) Bending of pipes, 7) Engineering, 8) Milling and turning of smaller parts, and 9) Project management. For each task, there were two to five partner candidates, some of which were candidates for several tasks.

Three selection criteria were given with the following priority: 1) minimize delay risks, 2) maximize earlier collaboration, and 3) minimize costs. The project had a tight schedule, thus minimization of risks was most important. Moreover, it was 
assumed that a successful collaboration history contributes to finishing the project in time. Costs were in this case the least important criterion.

Each partner candidate was given a probability distribution for finishing the tasks in time. Moreover, data on the candidates' collaboration history was readily available (see Figure 1), and the candidates' costs were estimated.

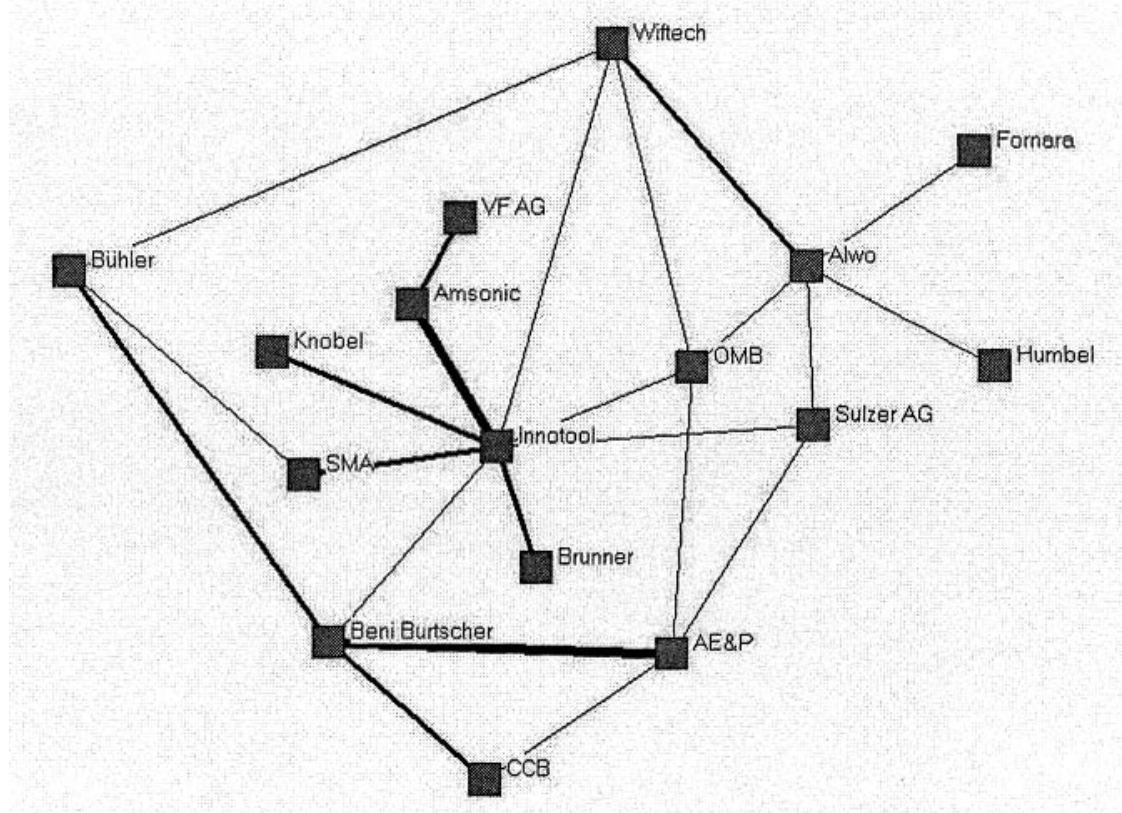

Figure 1 - Collaboration history of some members of Virtuelle Fabrik (line thickness corresponds to intensity)

\subsection{Case Analysis}

In the analysis of this case, fifteen Pareto-efficient configurations were identified using the optimization framework. Table 1 presents the performance of these configurations on the three selection criteria. The configurations have been sorted first by risk, second by collaboration, and third by cost, which was also the relative importance of the selection criteria. Moreover, Figure 2 illustrates this data in three dimensions.

The risk-measure used was the Expected Downside Risk (EDR, Eppen et al., 1989), where a smaller score is preferred to a greater one, zero being the theoretically best. The collaboration-score is calculated using a measure that accounts for earlier collaboration as well as for the total number of partners in a configuration. Also here a smaller score is preferred. Cost is the expected total cost in euros. 
Table 1 - Performance of fifteen Pareto-efficient configurations on three selection criteria

\begin{tabular}{rcl}
\hline \multicolumn{1}{c}{ Risk } & Collaboration & Cost (EUR) \\
\hline \multicolumn{1}{c}{ II 0,25} & 58 & 131312 \\
0,25 & 74 & 130795 \\
${ }^{2)} 0,75$ & 49 & 132116 \\
${ }^{3)} 0,75$ & 55 & 123215 \\
0,75 & 62 & 122710 \\
0,75 & 71 & 122698 \\
1,00 & 69 & 122131 \\
1,00 & 71 & 122119 \\
${ }^{4)} 1,25$ & 46 & 123215 \\
1,25 & 62 & 122057 \\
1,50 & 80 & 121702 \\
1,75 & 53 & 122057 \\
1,75 & 70 & 121640 \\
2,25 & 62 & 121640 \\
2,75 & 67 & 121628 \\
\hline
\end{tabular}

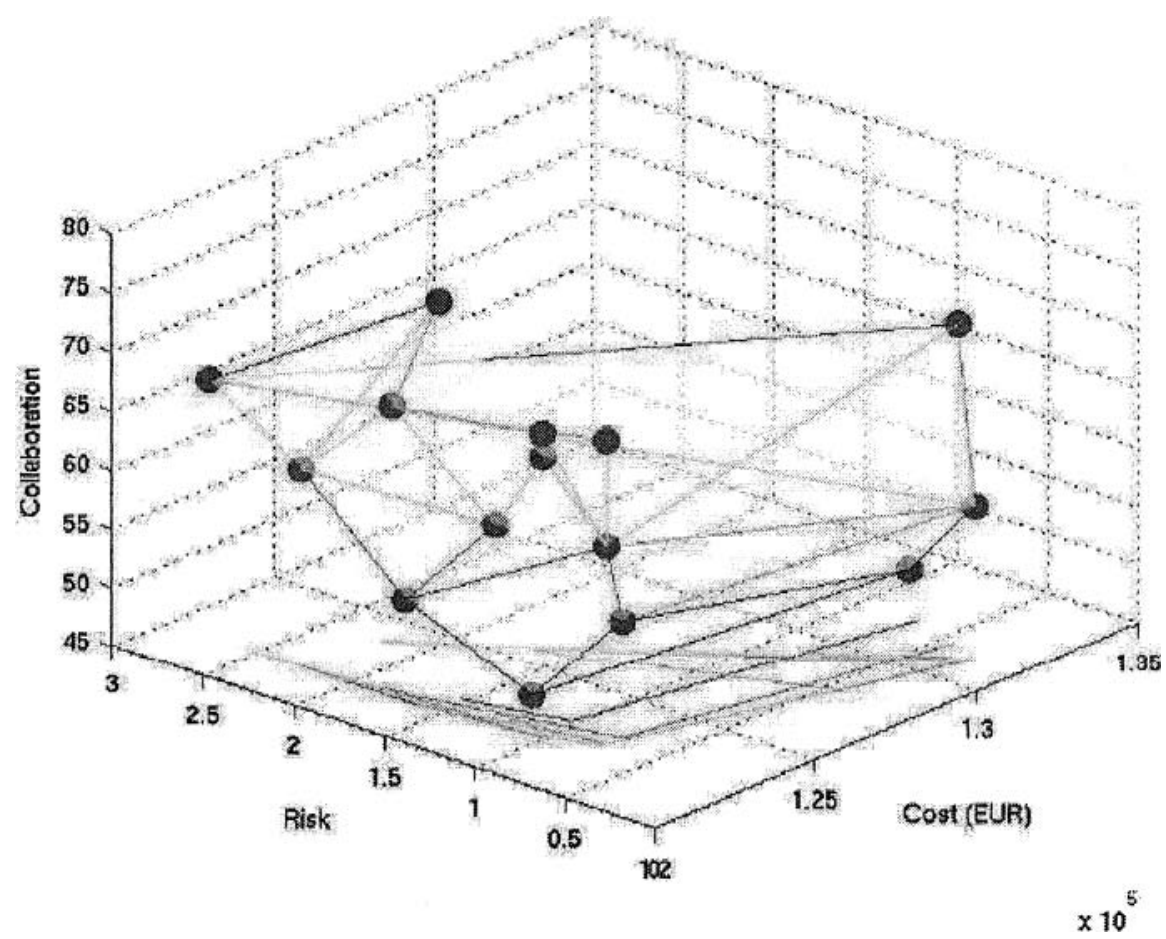

Figure 2 - The fifteen Pareto-efficient configurations in 3D 
With a closer look at Table 1 , one can identify at least four interesting configurations, labeled with superscripts $1-4$. Configuration 1) has significantly better collaboration-score than the other configuration with a low risk, but only a slightly higher cost. Configurations 2) and 3) have a higher risk than Configuration 1), but a better collaboration-score, or lower cost, respectively. Configuration 4), in turn, has a medium risk, but the overall best collaboration-score and a low cost.

After identifying the interesting configurations from Table 1, the DM can check which partners would be selected in each case. For instance, Table 2 shows the partners for each task in Configuration 1) as well as the partners that were actually chosen to the Magnetic Clutch Prototype project. Configuration 1) is selected for illustration purposes because its performance is in line with the preferences of the $D M$, i.e. risk minimization is most important. The computer-suggested configuration differs from the truly selected configuration in two tasks (boldface). The reason for this is that in the computer-suggestion the total number of partners is seven, which is two partners less than in the true configuration. However, the final decision was made manually based on the DM's good experiences with Amsonic and Fornara. The DM stressed that these two partners would be very reliable when it comes to accuracy.

Table 2 - Partners selected in Configuration 1) and in reality

\begin{tabular}{lll}
\hline Tasks of Magnetic Clutch Prototype & Configuration 1) & Truly selected \\
Grinding & Brunner & Brunner \\
Gear milling & Okey & Okey \\
Metal sheet forming & Beni Burtscher & Beni Burtscher \\
Milling and turning of bigger parts & SMA & SMA \\
Welding & Beni Burtscher & Amsonic \\
Bending of pipes & SMA & Fornara \\
Engineering & Schuler & Schuler \\
Milling and turning of smaller parts & Innotool & Innotool \\
Project management & VF AG & VF AG \\
\hline
\end{tabular}

\section{CONCLUSIONS}

This paper presents an application of a recently developed optimization framework for partner selection. We model partner selection as a centralised decision-making problem. This is reasonable if one entity is fully responsible for selecting the network partners. However, there are situations where the decision-making is in fact decentralised. This is the case if the partner candidates themselves decide with whom to collaborate. An example of a decentralised partner selection process is the formation inter-organisational research projects. In this case, the formation of the final consortium is a multi-party negotiation process between research teams at universities, research institutes, and companies.

Our model is potentially useful in cases where one DM selects network partners. Such cases occur in a VBE that repeatedly creates VOs whenever there is potential 
for value creation through collaboration. Customers often wish that only a single partner - the broker - is responsible for the operations of the VO. It is therefore natural that the broker has the control over the VO and the partner selection.

The centralised decision-making may lead to the following paradox. Consolidation of organisational competences allows the companies to focus on niche core-competences, which enables VOs to construct more complex products and services than an individual company could possibly do. However, the growth in the complexity of niche competences make also marketing and project management processes more complicated, since the broker or the project manager needs to manage not only the expertise of one's own company but also the expertise of other companies. The training of such multi-professionals is costly, which limits the possibilities of collaborative networks. Therefore, networking calls for efficient models of inter-organisational knowledge management.

In partner selection, different option-contracts are a potential topic for further studies. A portfolio consisting of option-contracts together with traditional contracts could be used to hedge against the risk of capacity shortfall. Here, the theoretical foundations can be drawn from portfolio selection methodology and real-options theory. Moreover, robust models for identifying poorly-performing partner candidates would be useful. The elimination of some candidates reduces the total number of potential configurations, which helps the selection.

\section{ACKNOWLEDGEMENTS}

The work has been co-funded by the European Commission through the Sixth Framework Programme Integrated Project ECOLEAD, as well as by the National Technology Agency of Finland (Tekes) and the National Workplace Development Programme (Tykes) through the COBTEC project.

\section{REFERENCES}

1. Camarinha-Matos LM, Afsarmanesh $\mathrm{H}$. Elements of a base VE infrastructure. Computers in Industry $2003 ; 51: 139-63$.

2. Camarinha-Matos LM, Afsarmanesh H. Collaborative Networks: A New Scientific Discipline. Joumal of Intelligent Manufacturing 2005; 16:439-52.

3. Degraeve, Z., Labro, E., Roodhooft, F. An Evaluation of Vendor Selection Models from a Total Cost of Ownership Perspective, European Joumal of Operational Research 2000;125:34-58.

4. Eppen GD, Martin RK, Schrage L. A Scenario Approach to Capacity Planning. Operations Research $1989 ; 37(4): 517-27$.

5. Gulati, R., Nohria, N., Zaheer, A. Strategic Networks. Strategic Management Journal 2000;21:203215.

6. Jarimo, T., Pulkkinen, U. A Multi-Criteria Mathematical Programming Model for Agile Virtual Organization Creation. Collaborative Networks and their Breeding Environments, PRO-VE:05 Sixth IFIP Working Conference on VIRTUAL ENTERPRISES, 26-28 September 2005, Valencia, Spain, editors L. M. Camarinha-Matos, H. Afsarmanesh, A. Ortiz, Springer, 2005:127-134.

7. Jarimo, T., Salo, A. Optimal Partner Selection in Virtual Organisations with Capacity Risk and Network Interdependencies. Submitted Manuscript: 2006.

8. Keeney, R. L., Raiffa, H. Decisions with Multiple Objectives; Preferences and Value Tradeoffs. Cambridge University Press, 1993.

9. Lau, H. C.W., Wong, E. T. T. Partner selection and information infrastructure of a virtual enterprise network. International Joumal of Computer Integrated Manufacturing 2001;14(2):186-193. 
10. Taha, H. A. Operations Research: An Introduction. Prentice-Hall Intemational, 1997.

11. Tølle, M., Bernus, $P$. Reference models supporting enterprise networks and virtual enterprises. International Journal of Networking and Virtual Organisations 2003;2(1):2-15. 